\title{
A SOLUTION TO THE SPLITTING MIXED GROUP PROBLEM OF BAER
}

\author{
BY \\ PHILLIP GRIFFITH $\left({ }^{1}\right)$
}

1. Introduction. All groups considered in this paper are assumed to be additively written abelian groups. We follow for the most part the notation and terminology of [8]. We mention the following notations and definitions. If $G$ is an abelian group, then $t G$ denotes the maximal torsion subgroup of $G$ and $G_{p}$ denotes the primary component of $t G$ for the prime $p$. The $p$-height of an element $x \in G$ is denoted by $h_{p}^{G}(x)$. We call a group $G$ Hausdorff if it is Hausdorff in its $n$-adic topology. The group of rationals is denoted by $Q$ and the subgroup of $Q$ consisting of the integers by $Z$. The direct product of $\aleph_{0}$ copies of $Z$ is denoted by $P$. After Nunke [11], a group $G$ is called locally free if it is isomorphic to a pure subgroup of a direct product of copies of $Z$, that is, if $G$ is $\boldsymbol{N}_{1}$-free and separable.

The central problem of this paper is one posed by Baer [1] in 1936 (also see Problem 30 in [8]) that asks for a characterization of those groups $G$ for which $\operatorname{Ext}(G, T)=0$ for all torsion groups $T$. Such a group is called a Baer group or more simply a $B$-group. Baer [1] proved that any countable $B$-group is free. Since subgroups of $B$-groups are again $B$-groups, it follows that $B$-groups are $\aleph_{1}$-free. More recently Baer [3], Erdös [7] and Sasiada [8] independently proved that $P$ is not a $B$-group. J. Rotman [12] showed that separable $B$-groups are slender and Nunke [11] removed the condition of separability. In this paper we settle the problem by showing that $B$-groups are necessarily free. More generally, for a nonempty subset $N$ of the primes, we call a group $G$ a $B^{N}$-group if $\operatorname{Ext}(G, T)=0$ for all torsion groups $T$ such that $T_{p}=0$ for $p \notin N$. We are successful in finding necessary and sufficient conditions on the structure of a group $G$ in order that $G$ be a $B^{N}$-group for a prescribed subset $N$ of the primes. The proofs of these results rest on the existence of a remarkable class of mixed groups. We show, for any cardinal number $\mu$, that there is a Hausdorff mixed group $M$ such that $M / t M$ is divisible of rank $\mu$ and such that every torsion free subgroup of $M$ is free. Our method of constructing such a group $M$ is a somewhat complicated one. The most important tool used in showing that every torsion free subgroup of $M$ is free is the "back-and forth" technique for direct sum decompositions introduced by Hill and Megibben in [10]. The final section of this paper deals with locally free $B^{N}$-groups (not all

Presented to the Society, November 25, 1967 under the title Baer groups are free; received by the editors June 22, 1967.

( $\left.{ }^{1}\right)$ The author wishes to acknowledge support by the National Aeronautics and Space Administration Grant NsG(T)-52. 
$B^{N}$-groups need be locally free). We show that if $N$ is a nonempty proper subset of the primes, then there is a locally free $B^{N}$-group that is not free.

2. An existence theorem concerning mixed groups. We begin by establishing a special case of our main result on mixed groups.

LEMMA 2.1. There is a Hausdorff mixed group $X$ such that $X / t X \cong Q$ and such that any torsion free subgroup of $X$ is cyclic.

Proof. For each prime $p$, let $M^{(p)}=\prod_{n<\omega}\left\{b_{p n}\right\}$ where $\left\{b_{p n}\right\}$ denotes the cyclic group of order $p^{n}$ and set $M=\prod_{p} M^{(p)}$ where $p$ ranges over the primes. Let $u$ $=\left\langle u_{p}\right\rangle \in M$ where $u_{p}$ is an element of $M^{(p)}$ defined as follows: $u_{p}=\left\langle p^{e_{n}} b_{p n}\right\rangle$ where $e_{n}=[n / 2]$ ([ ] denotes the greatest integer function). Note that $h_{p}^{M(p)}\left(u_{p}\right)=0$ since $e_{1}=0$. It is elementary to see that $u_{p}$ is an element of infinite order in $M^{(p)}$ and hence that $u$ is an element of infinite order in $M$. It is also easily seen, for each $y=\left\langle y_{p}\right\rangle$ $\in t M$ and each prime $p$, that $h_{p}^{M}(n u+y)=h_{p}^{M(p)}\left(n u_{p}+y_{p}\right)<\infty$ where $n$ is a nonzero integer. To show that $h_{p}^{(M / t M)}(u+t M)=\infty$ for each prime $p$, it suffices to show that for each nonzero integer $m$ and each prime $p$ there is an element $x^{(m)} \in t M^{(p)}$ such that $u_{p}-x_{p}^{(m)} \in p^{m} M^{(p)}$. Clearly, there is a positive integer $N$ such that if $n \geqq N$ then $e_{n} \geqq m$. Define $x_{p}^{(m)}=\left\langle\delta_{n} b_{p n}\right\rangle$ where $\delta_{n}=p^{e_{n}}$ for $n=1, \ldots, N$ and $\delta_{n}=0$ for $n>N$. Then $u_{p}-x_{p}^{(m)} \in p^{m} M^{(p)}$ and $x_{p}^{(m)} \in t M^{(p)}$. Since $M / t M$ is torsion free there is a pure subgroup $X$ of $M$ containing $t M$ and $u$ such that $X / t M$ is a pure rank one subgroup of $M / t M$. Since rank $(X / t M)=1$ and since $h_{p}^{(X / t M)}(u+t M)=h_{p}^{(M / t M)}(u+t M)=\infty$ for each $p$, it follows that $X / t M=X / t X \cong Q$.

Suppose that $A$ is a subgroup of $X$ such that $A \cap t X=0$. We may further suppose that $A \neq 0$. Since $A \cong\{A, t X\} / t X \subseteq X / t X \cong Q$, it follows that $A$ is torsion free of rank one. Let $a \neq 0 \in A$. Since $X / t X \cong Q$ there are nonzero integers $r$ and $s$ such that $r a=s u+y$ where $y=\left\langle y_{p}\right\rangle \in t X$. We have already observed that $h_{p}^{X}(r a)=h_{p}^{X}(s u+y)$ $=h_{p}^{M}(s u+y)<\infty$ for each $p$. Hence $h_{p}^{A}(r a)<\infty$ for each prime $p$. There is a positive integer $N$ such that $y_{p}=0$ for all $p>N$. Let $p$ be any prime larger than $N+|s|$. Then $(s, p)=1$ and $h_{p}^{A}(r a) \leqq h_{p}^{X}(s u+y)=h_{p}^{M(p)}\left(s u_{p}\right)$. Since $s$ and $p$ are relatively prime, $h_{p}^{M(p)}\left(s u_{p}\right)=h_{p}^{M(p)}\left(u_{p}\right)=0$. Thus, $h_{p}^{A}(r a)=0$ for each prime $p$ larger than $N+|s|$. Hence, the height sequence for $r a$ in $A$ is zero except for a finite number of primes and contains no "infinities". (For definition of height sequence, see [8].) It follows by a Theorem of Baer [2] that $A \cong Z$.

Let $X$ be a group satisfying Lemma $1, \mu$ a cardinal number and let $\Lambda$ be an initial segment of the ordinal numbers having cardinality $\mu$. For the remainder of this section, we define the group $K_{\mu}$ to be $\sum_{\lambda \in \Lambda} X_{\lambda}$ where $X_{\lambda} \cong X$ for each $\lambda \in \Lambda$. We now improve Lemma 2.1 .

LemMA 2.2. Let $A$ be a torsion free subgroup of $K_{\mu}$. Then $A$ is $\aleph_{1}$-free.

Proof. By a theorem of Pontryagin [8] we need only show that every subgroup of $A$ of finite rank is free. Since any subgroup of $A$ of finite rank is isomorphic to a torsion free group contained in a finite number of the groups $X_{\lambda}$, it is enough to 
prove the lemma for $K_{n}$ where $n$ is a positive integer. For $n=1$, the lemma follows from Lemma 2.1. Therefore, suppose the result holds for all integers $\mu \leqq n$ and consider a torsion free subgroup $A$ of $K_{n+1}=\sum_{i=1}^{n+1} X_{i}$. Let $\theta$ be the natural projection of $K_{n+1}$ onto $K_{n+1} / t K_{n+1}$ where $\theta$ restricted to $X_{i}$ is the natural projection of $X_{i}$ onto $X_{i} / t X_{i}$. Also let $B=\left\{a \in A \mid \theta(a) \in \sum_{i=1}^{n} \theta\left(X_{i}\right)\right\}$ and let $\pi$ be the natural projection of $K_{n+1}$ onto $\sum_{i=1}^{n} X_{i}$. Suppose that $b \in B \cap \operatorname{Ker} \pi=B \cap X_{n+1}$. Since $\theta(b) \in \sum_{i=1}^{n} \theta\left(X_{i}\right)$, it follows that $b \in t X_{n+1} \subseteq t K_{n+1}$. Therefore, $b=0$ and $B \cong \pi(B)$ $\subseteq \sum_{i=1}^{n} X_{i} \cong K_{n}$. It follows from the induction hypothesis that $B$ is free. Hence, $B=\sum_{j=1}^{k}\left\{b_{j}+y_{j}\right\}$ where $k \leqq n, \quad b_{j} \in \sum_{i=1}^{n} X_{i}$ and $y_{j} \in X_{n+1}$. Since $\theta\left(b_{j}+y_{j}\right)$ $\in \sum_{i=1}^{n} \theta\left(X_{i}\right)$, it follows that $y_{j} \in t X_{n+1}$ for $j=1, \ldots, k$. Let $m$ be a positive integer such that $m y_{j}=0$ for $j=1, \ldots, k$ and let $\rho$ be the natural projection of $K_{n+1}$ onto $X_{n+1}$. Set $\phi=m \rho$. It is easily verified that $A \cap \operatorname{Ker} \phi=B$ and that $\phi(A)$ is a torsion free subgroup of $X_{n+1}$. By Lemma $2.1, \phi(A)$ is cyclic. Hence $A \cong B+\phi(A)$ and thus $A$ is free.

We now establish the main result of this section. For notational convenience we use the notation $\sum_{I} X_{\lambda}$ to indicate $\sum_{\lambda \in I} X_{\lambda}$ where $I \subseteq \Lambda$.

THEOREM 2.3. For any cardinal number $\mu$, there is a Hausdorff mixed group $M$ such that $M / t M$ is divisible of rank $\mu$ and such that every torsion free subgroup of $M$ is free.

Proof. Let $M=K_{\mu}=\sum_{\Lambda} X_{\lambda}$. For the purpose of this proof, we assume that $0 \in \Lambda$ and that $X_{0}^{\prime}=0$. From the definition of $K_{\mu}$, it is enough to show that every torsion free subgroup of $K_{\mu}$ is free. If $\mu$ is countable, the result follows from Lemma 2.2. Hence, we may assume that $\mu$ is uncountable. Let $A$ be a torsion free subgroup of $K_{\mu}$. Since $\left|K_{\mu} / t K_{\mu}\right|=\mu=|\Lambda|$, it follows that $|A| \leqq|\Lambda|$. Thus, we may label the elements of $A$ with the ordinals in $\Lambda$ starting with $a_{0}=0 \in A$ (we label $0 \in A$ repeatedly if necessary). Again let $\theta$ be the natural projection of $K_{\mu}$ onto $K_{\mu} / t K_{\mu}$. We wish to express $A$ and a subset of $\Lambda$ as unions of well-ordered monotone sequences $\left[A_{\alpha}\right]_{\alpha \in \Lambda}$ and $\left[I_{\alpha}\right]_{\alpha \in \Lambda}$, respectively such that $A_{0}=\left\{a_{0}\right\}=0$ and $I_{0}=[0]$ and such that

(i) $\left|I_{\alpha+1}-I_{\alpha}\right| \leqq \aleph_{0}$.

(ii) $I_{\alpha}=\bigcup_{\gamma<\alpha} I_{\gamma}$ and $A_{\alpha}=\bigcup_{\gamma<\alpha} A_{\gamma}$ if $\alpha$ is a limit ordinal.

(iii) $a_{\alpha} \in A_{\alpha+1}$.

(iv) $A_{\alpha}=A \cap\left(\sum_{I_{\alpha}} X_{\lambda}\right)=\left\{a \in A \mid \theta(a) \in \sum_{I_{\alpha}} \theta\left(X_{\lambda}\right)\right\}$.

Suppose that the $I_{\alpha}$ 's and the $A_{\alpha}$ 's satisfying (i)-(iv) have been chosen for all $\alpha<\beta, \beta \in \Lambda$. If $\beta$ is a limit ordinal, then we need only set $I_{\beta}=\bigcup_{\alpha<\beta} I_{\alpha}$ and $A_{\beta}$ $=\bigcup_{\alpha<\beta} A_{\alpha}$. We may assume that $\beta-1$ exists. We define sequences $\left[B_{n}\right]_{n<\omega}$ and $\left[L_{n}\right]_{n<\omega}$ inductively as follows: $B_{1}=\left\{A_{\beta-1}, a_{\beta-1}\right\}$ and let $L_{1}$ be the smallest subset of $\Lambda$ such that $B_{1} \subseteq \sum_{L_{1}} X_{\lambda}$. Clearly, $I_{\beta-1} \subseteq L_{1}$ and $\left|L_{1}-I_{\beta-1}\right| \leqq \boldsymbol{\aleph}_{0}$. In general for $n>1$, we let $L_{n}$ be the smallest subset of $\Lambda$ such that $B_{n}=\left\{a \in A \mid \theta(a) \in \sum_{L_{n-1}} \theta\left(X_{\lambda}\right)\right\}$ $\subseteq \sum_{L_{n}} X_{\lambda}$. Since $B_{n+1}=\left\{a \in A \mid \theta(a) \in \sum_{L_{n}} \theta\left(X_{\lambda}\right)\right\}$ and since $B_{n} \subseteq \sum_{L_{n}} X_{\lambda}$, it follows that $B_{n} \subseteq B_{n+1}$ and that $L_{n} \subseteq L_{n+1}$. Set $I_{\beta}=\bigcup_{n<\omega} L_{n}$ and set $A_{\beta}=\bigcup_{n<\omega} B_{n}$. Since 
(ii) and (iii) clearly hold for $\left[I_{\alpha}\right]_{\alpha \leqq \beta}$ and $\left[A_{\alpha}\right]_{\alpha \leqq \beta}$, we need only verify (i) and (iv). If $a_{\beta-1} \in A_{\beta-1}$, it is easily seen that $A_{\beta}=A_{\beta-1}$ and $I_{\beta}=I_{\beta-1}$. Therefore, we may assume that $a_{\beta-1} \notin A_{\beta-1}$. To show that $\left|I_{\beta}-I_{\beta-1}\right| \leqq \aleph_{0}$, it suffices to show that $\left|L_{n}-I_{\beta-1}\right| \leqq \aleph_{0}$ for each $n$. Since we have already observed that $\left|L_{1}-I_{\beta-1}\right| \leqq \boldsymbol{\aleph}_{0}$, we suppose that $\left|L_{n}-I_{\beta-1}\right| \leqq \aleph_{0}$ and consider $L_{n+1}$. By definition of $\dot{L}_{n+1}$, it is enough to show that $\left|B_{n+1} / A_{\beta-1}\right| \leqq \aleph_{0}$. We also may assume that $L_{n} \neq L_{n+1}$. Let $\pi$ be the natural projection of $K_{\mu}$ onto $\sum_{L_{n}-I_{\beta-1}} X_{\lambda}$. Clearly, $A_{\beta-1} \subseteq \operatorname{Ker} \pi \cap B_{n+1}$. Suppose that $x \in B_{n+1}$ and that $m x \in \operatorname{Ker} \pi \cap B_{n+1}$ where $m$ is a nonzero integer. Then $x=y+w$ where $y \in \sum_{I_{\beta-1}} X_{\lambda}$ and $w \in \sum_{L_{n+1}-I_{\beta}-1} X_{\lambda}$. Since $\pi(w) \in t\left(\sum_{L_{n-I_{\beta}-1}} X_{\lambda}\right)$ and since $\theta(x) \in \sum_{L_{n}} \theta\left(X_{\lambda}\right)$, we have that $w \in t\left(\sum_{L_{n+1}-I_{\beta-1}} X_{\lambda}\right)$ which implies that $\theta(x)=\theta(y) \in \sum_{I_{\beta-1}} \theta\left(X_{\lambda}\right)$. By (iv), $x \in A_{\beta-1}$. Hence, $\operatorname{Ker} \pi \cap B_{n+1}=A_{\beta-1}$ and $\pi\left(B_{n+1}\right)$ is torsion free. Since $\left|L_{n}-I_{\beta-1}\right| \leqq \mathcal{\aleph}_{0}$, it follows from the definition of the $X_{\lambda}$ 's that $\left|\pi\left(B_{n+1}\right)\right| \leqq \boldsymbol{\aleph}_{0}$. Thus $\left|B_{n+1} / A_{\beta-1}\right| \leqq \boldsymbol{\aleph}_{0}$ and hence $\left|L_{n+1}-I_{\beta-1}\right| \leqq \boldsymbol{\aleph}_{0}$. Thus (i) holds for $\alpha \leqq \beta$. Now if $x \in A \cap\left(\sum_{I_{\beta}} X_{\lambda}\right)$ then $x \in A \cap\left(\sum_{L_{n}} X_{\lambda}\right)$ for some $n$. Therefore, $\theta(x) \in \sum_{L_{n}} \theta\left(X_{\lambda}\right)$ which implies that $x \in B_{n+1} \subseteq A_{\beta}$. Since $A_{\beta} \subseteq A \cap$ $\left(\sum_{I_{\beta}} X_{\lambda}\right)$, we have that $A_{\beta}=A \cap\left(\sum_{I_{\beta}} X_{\lambda}\right)$. We also have that $A_{\beta}=A \cap\left(\sum_{I_{\beta}} X_{\lambda}\right)$ $\subseteq\left\{a \in A \mid \theta(a) \in \sum_{I_{\beta}} \theta\left(X_{\lambda}\right)\right\}$. If $\theta(a) \in \sum_{I_{\beta}} \theta\left(X_{\lambda}\right)$ where $a \in A$, then $\theta(a) \in \sum_{L_{n}} \theta\left(X_{\lambda}\right)$ for some $n$. By definition, $a \in B_{n+1} \subseteq A_{\beta}$. Hence,

$$
A_{\beta}=A \cap\left(\sum_{I_{\beta}} X_{\lambda}\right)=\left\{a \in A \mid \theta(a) \in \sum_{I_{\beta}} \theta\left(X_{\lambda}\right)\right\} \text {. }
$$

Thus $\left[A_{\alpha}\right]_{\alpha \leqq \beta}$ and $\left[I_{\alpha}\right]_{\alpha \leqq \beta}$ satisfy (i)-(iv).

We now establish

(v) $A_{\alpha}$ is a direct summand of $A_{\alpha+1}$ and $A_{\alpha+1}=A_{\alpha}+F_{\alpha}$ where $F_{\alpha}$ is free.

Let $\pi_{\alpha}$ be the natural projection of $K_{\mu}$ onto $\sum_{I_{\alpha+1}-I_{\alpha}} X_{\lambda}$ (we may assume that $\left.I_{\alpha+1} \neq I_{\alpha}\right)$. Suppose that $x \in A_{\alpha+1}$ such that $\pi(x) \in t\left(\sum_{I_{\alpha+1}-I_{\alpha}} X_{\lambda}\right)$. Then $x=y+w$ where $y \in \sum_{I_{\alpha}} X_{\lambda}$ and where $w \in t\left(\sum_{I_{\alpha+1}-I_{\alpha}} X_{\lambda}\right)$. This implies that $\theta(x)=\theta(y)$ $\in \sum_{I_{\alpha}} \theta\left(X_{\lambda}\right)$ which implies by (iv) that $x \in A_{\alpha}$. Hence, $A_{\alpha+1} \cap \operatorname{Ker} \pi_{\alpha}=A_{\alpha+1}$ $\cap\left(\sum_{I_{\alpha}} X_{\lambda}\right)=A \cap\left(\sum_{I_{\alpha}} X_{\lambda}\right)=A_{\alpha}$ and $\pi_{\alpha}\left(A_{\alpha+1}\right)$ is torsion free. Therefore, $A_{\alpha+1} / A_{\alpha}$ is isomorphic to a torsion free subgroup of $\sum_{I_{\alpha+1}-I_{\alpha}} X_{\lambda}$. Since by (i) $\left|I_{\alpha+1}-I_{\alpha}\right| \leqq \boldsymbol{\aleph}_{0}$ and since $\left|K_{\aleph_{0}} / t K_{\aleph_{0}}\right| \leqq \boldsymbol{\aleph}_{0}$, it follows that $\left|A_{\alpha+1} / A_{\alpha}\right| \leqq \boldsymbol{\aleph}_{0}$. By Lemma $2, A_{\alpha+1} / A_{\alpha}$ is free. Thus (v) is established. Since $A_{0}$ is free, then (ii) and (v) imply that $A$ is free.

3. The structure of $B^{N}$-groups. Let $G$ be a torsion free group and let $T$ be a torsion group. Then an extension $T \longleftrightarrow H \rightarrow G$ is called a quasi-splitting extension of $T$ by $G$ if $H$ is quasi-isomorphic to $T+G$. By a Theorem of C. Walker [14], the extension above is quasi-splitting if and only if it represents an element of finite order in Ext $(G, T)$. The following theorem characterizes those torsion free groups $G$ for which every extension $T \longleftrightarrow H \rightarrow G$ is quasi-splitting for all torsion groups $T$.

THEOREM 3.1. Let $G$ be a torsion free group. Then $\operatorname{Ext}(G, T)$ is torsion for all torsion groups $T$ if and only if $G$ is free.

Proof. The sufficiency is clear. Therefore, suppose that $G$ is a torsion free group such that $\operatorname{Ext}(G, T)$ is torsion for all torsion groups $T$. Let $\mu=\operatorname{rank}(G)$ and let $M$ 
be a group satisfying Theorem 2.3 such that $\operatorname{rank}(M / t M)=\mu$. Since $M / t M$ is torsion free and divisible there is a monomorphism $f: G \succ M / t M$. Let $\theta$ be the natural map of $M$ onto $M / t M$. The exactness of the sequence

$$
\operatorname{Hom}(G, M) \stackrel{\theta_{*}}{\rightarrow} \operatorname{Hom}(G, M / t M) \stackrel{\delta_{G}}{\longrightarrow} \operatorname{Ext}(G, t M)
$$

implies that there is a nonzero integer $n$ such that $n f \in \operatorname{Im} \theta_{*}$, that is, there is a homomorphism $\phi \in \operatorname{Hom}(G, M)$ such that $n f=\theta \phi$. Since $n f$ is also a monomorphism, $\phi$ must be a monomorphism and $\phi(G) \cap \operatorname{Ker} \theta=\phi(G) \cap t M=0$. Thus $G$ is isomorphic to a torsion free subgroup of $M$ which implies by Theorem 2.3 that $G$ is free.

The following corollary settles the question of Baer that was mentioned in the introduction.

Corollary 3.2. A group $G$ is a Baer group if and only if it is free.

Proof. Again the sufficiency is clear. Since Baer groups are necessarily torsion free (see [1]) we may apply Theorem 3.1 to prove the necessity.

Before continuing, one should observe that our results are valid for modules over a principal ideal domain $\left({ }^{2}\right)$. Let $N$ be a nonempty subset of the primes and let $I_{N}$ be the subring of the rationals $Q$ defined by the rule: $m / n \in Q$, where $m, n \in Z$ and $n \neq 0$, is an element of $I_{N}$ if and only if $n$ and $p$ are relatively prime for each prime $p \in N$. We also use the symbol $I_{N}$ to denote the additive group of $I_{N}$. However, no confusion should arise. Observe that a torsion group $T$, where $T_{p}=0$ for $p \notin N$, can be considered a module in a natural fashion over the ring $I_{N}$. For a group $G$, one should also observe that $\operatorname{Hom}\left(I_{N} \otimes G, T\right)=\operatorname{Hom}_{I_{N}}\left(I_{N} \otimes G, T\right)\left({ }^{3}\right)$. We now establish the following lemma.

LeMma 3.3. Let $N$ be a nonempty subset of the primes and let $T$ be a torsion group such that $T_{p}=0$ for $p \notin N$. If $G$ is a group such that $\operatorname{Tor}\left(G, I_{N} / Z\right)=0$, then $\operatorname{Ext}(G, T)$, $\operatorname{Ext}\left(I_{N} \otimes G, T\right)$ and $\operatorname{Ext}_{I_{N}}\left(I_{N} \otimes G, T\right)$ are isomorphic as abelian groups.

Proof. We may assume that $N$ is a proper subset of the primes since otherwise $I_{N}=Z$. Let $\tilde{N}$ be the set of primes not in $N$. From the definition of $I_{N}$ we obtain the exact sequence $Z \succ I_{N} \rightarrow \sum_{p \in \tilde{N}} C\left(p^{\infty}\right)$ which yields the exact sequence $Z \otimes G \succ I_{N} \otimes G \rightarrow \sum_{p \in \mathbb{N}} C\left(p^{\infty}\right) \otimes G$. Hence, we obtain the exact cohomology sequence $\operatorname{Ext}\left(\sum_{p \in \mathbb{N}} C\left(p^{\infty}\right) \otimes G, T\right) \longmapsto \operatorname{Ext}\left(I_{N} \otimes G, T\right) \rightarrow \operatorname{Ext}(Z \otimes G, T)$. Since $\sum_{p \in \mathbb{N}} C\left(p^{\infty}\right) \otimes G$ and $T$ are torsion groups with no nonzero primary components in common, we have that $\operatorname{Ext}\left(\sum_{p \in \mathbb{N}} C\left(p^{\infty}\right) \otimes G, T\right)=0$ and thus

$$
\operatorname{Ext}\left(I_{N} \otimes G, T\right) \cong \operatorname{Ext}(Z \otimes G, T) \cong \operatorname{Ext}(G, T) .
$$

( $\left.{ }^{2}\right)$ All rings considered in this paper are assumed to be commutative with identity and all modules are assumed to be unital. $R=Z$.

${ }^{(3)}$ We drop the subscript " $R$ " on the functors $\operatorname{Hom}_{R}(A, B)$ and $\operatorname{Ext}_{R}(A, B)$ only when 
Let $E$ be the $I_{N}$-injective envelope of $T$. Clearly $E$ is both injective as an $I_{N^{-}}$ module and as an abelian group. Therefore, the exactness of $T \longrightarrow E \rightarrow E / T$ induces exactness of the rows of the commutative diagram:

$\operatorname{Hom}\left(I_{N} \otimes G, T\right) \rightarrow \operatorname{Hom}\left(I_{N} \otimes G, E\right) \rightarrow \operatorname{Hom}\left(I_{N} \otimes G, E / T\right) \rightarrow \operatorname{Ext}\left(I_{N} \otimes G, T\right)$

$\operatorname{Hom}_{I_{N}}\left(I_{N} \otimes G, T\right) \rightarrow \operatorname{Hom}_{I_{N}}\left(I_{N} \otimes G, E\right) \rightarrow \operatorname{Hom}_{I_{N}}\left(I_{N} \otimes G, E / T\right) \rightarrow \operatorname{Ext}_{I_{N}}\left(I_{N} \otimes G, T\right)$

Thus the cokernels are isomorphic.

THEOREM 3.4. A group $G$ is a $B^{N}$-group if and only if $I_{N} \otimes G$ is free as an $I_{N^{-}}$ module.

Proof. Suppose that $G$ is a $B^{N}$-group. Then clearly $(t G)_{p}=0$ for each $p \in N$. Therefore, $I_{N} \otimes(t G)=0$ and hence $I_{N} \otimes G \cong I_{N} \otimes(G / t G)$. The exactness of $0=\operatorname{Hom}(t G, T) \rightarrow \operatorname{Ext}(G / t G, T) \rightarrow \operatorname{Ext}(G, T)=0$, when $T$ is a torsion group such that $T_{p}=0$ for $p \notin N$, implies that $G / t G$ is a $B^{N}$-group. By Lemma 3.3, $I_{N}$ $\otimes(G / t G)$ is a Baer module for the ring $I_{N}$. Hence, $I_{N} \otimes(G / t G)$ is free as an $I_{N^{-}}$ module and thus $I_{N} \otimes G$ is also free as an $I_{N}$-module. With the aid of Lemma 3.3 the sufficiency is easily obtained.

A corollary to Theorem 3.4 is the following:

Corollary 3.5. $G$ is a $B^{N}$-group if and only if $(t G)_{p}=0$ for each $p \in N$ and $G / t G$ is isomorphic to a subgroup of $\sum_{\mu} I_{N}$ where $\mu=\operatorname{rank}(G / t G)$.

Proof. By Theorem 3.4, $G$ is a $B^{N}$-group if and only if $I_{N} \otimes G \cong \sum_{\mu} I_{N}$ for some cardinal number $\mu$. Therefore, $I_{N} \otimes(t G)=0$, which holds if and only if $(t G)_{p}=0$ for $p \in N$. Hence $I_{N} \otimes G \cong I_{N} \otimes(G / t G)$. Fiom the exactness of $Z \gg I_{N} \rightarrow I_{N} / Z$, we obtain $G / t G \cong Z \otimes(G / t G) \longrightarrow I_{N} \otimes(G / t G) \rightarrow\left(I_{N} / Z\right) \otimes(G / t G)$. Since $\left(I_{N} / Z\right) \otimes$ $(G / t G)$ is torsion, we have that $\mu=\operatorname{rank}\left(I_{N} \otimes(G / t G)\right)=\operatorname{rank}(G / t G)$.

Our next corollary is an immediate consequence of Corollary 3.5 and a Theorem of Nunke [11] on slender groups.

Corollary 3.6. A torsion free $B^{N}$-group is slender.

4. On locally free $B^{N}$-groups. Let $N$ be a nonempty proper subset of the primes. Although Theorem 3.4 implies there are nonfree $B^{N}$-groups (for example the group $I_{N}$ ), one might suspect that locally free $B^{N}$-groups are free. However, we shall presently show, for each nonempty proper subset $N$ of the primes, that there is a pure subgroup of $P$ and hence a locally free group which is a nonfree $B^{N}$-group. We begin by first generalizing a method of Chase [5] for constructing pure subgroups of $P$ with certain prescribed properties. Let $S$ denote the group of finite sequences in $P$ and, for the purposes of our next lemma and theorem, let $E$ denote the cotorsion completion of $S$. (For information concerning cotorsion groups, see [9].) 
LEMMA 4.1. Let $C$ be a countable pure subgroup of $P$ that contains $S$ and let $U$ be a pure subgroup of $E$. Then there is a pure subgroup $A$ of $P$ such that $A$ contains $C$ and $A / C \cong U$.

Proof. Specker [13] has shown that $P$ contains a pure free subgroup of rank $\boldsymbol{\aleph}_{1}$. It follows by a Theorem of Chase [6] on pure independence, that any maximal pure independent subset of $P$ has cardinality at least $\boldsymbol{\aleph}_{1}$. Hence, there must be a subgroup $F$ of $P$ such that $C \subseteq F$ and such that $F / C$ is a pure free subgroup of $P / C$ of rank $\aleph_{0}$. Since $P / S$ is cotorsion (see [11]) and since $C$ is a pure subgroup of $P$ containing $S$, then $P / C$ is also a torsion free cotorsion group. Therefore, $P / C$ $=K / C+\bar{C} / C$ where $K / C$ is Hausdorff, $F \subseteq K$ and $\bar{C} / C$ is divisible. Let $H$ be the subgroup of $P$ such that $H / C$ is the $n$-adic closure of the pure free subgroup $F / C$ in $K / C$. Since $(K / C) /(H / C)$ must be reduced and torsion free, it follows that $H / C$ is cotorsion and that $H / C$ is a direct summand of $K / C$. Since $F / C$ is pure and dense in $H / C$, we have that $H / C \cong E$. Therefore, the group $U$ may be identified with a pure subgroup of $P / C$. Thus, there is a pure subgroup $A$ of $P$ such that $A$ contains $C$ and $A / C \cong U$.

THEOREM 4.2. Let $\left[U_{\alpha}\right]_{\alpha<\Omega}$ be a family of countable pure subgroups of $E$. Then there is a pure subgroup $A$ of $P$ such that $A=\bigcup_{\alpha<\Omega} A_{\alpha}$ where the subgroups $A_{\alpha}$ satisfy:

(i) If $\alpha<\beta, A_{\alpha} \subseteq A_{\beta}$.

(ii) $A_{\alpha}$ is free of rank $\aleph_{0}$.

(iii) $A_{\alpha+1} / A_{\alpha} \cong U_{\alpha}$.

(iv) If $\alpha$ is a limit ordinal, $A_{\alpha}=\bigcup_{\beta<\alpha} A_{\beta}$.

Furthermore, if there is a countable ordinal $\lambda$ such that $U_{\alpha} \neq 0$ and $\operatorname{Hom}\left(U_{\alpha}, Z\right)=0$ for all $\alpha>\lambda$, then $A$ is not free.

Proof. The construction of a pure subgroup $A$ of $P$ satisfying (i)-(iv) is an immediate consequence of Lemma 4.1. Hence, suppose that there is a countable ordinal $\lambda$ such that $U_{\alpha} \neq 0$ and $\operatorname{Hom}\left(U_{\alpha}, Z\right)=0$ for all $\alpha>\lambda$. Suppose that $f \neq 0$ $\in \operatorname{Hom}(A, Z)$ such that $f\left(A_{\lambda+1}\right)=0$. Let $\alpha$ be the smallest ordinal such that $f\left(A_{\alpha}\right)$ $\neq 0$. Clearly, $\alpha$ cannot be a limit ordinal. Hence, $\alpha=\beta+1$ and $\beta \geqq \lambda+1$ since $f\left(A_{\lambda+1}\right)=0$. Let $h$ be the restriction of $f$ to $A_{\beta+1}$. Since $h \neq 0$ and since $h\left(A_{\beta}\right)=0$, it follows that there is a nonzero homomorphism in $\operatorname{Hom}\left(A_{\beta+1} / A_{\beta}, Z\right) \cong$ Hom $\left(U_{\beta}, Z\right)$. But this is a contradiction since $\beta \geqq \lambda+1>\lambda$. Thus, if $f \in \operatorname{Hom}(A, Z)$ and $f\left(A_{\lambda+1}\right)=0$ then $f=0$, that is, Hom $\left(A / A_{\lambda+1}, Z\right)=0$. Since $U_{\alpha} \neq 0$ for all $\alpha>\lambda$, we have that $|A|=\aleph_{1}$. Clearly, no free group $F$ of cardinality $\aleph_{1}$ has the property that $\operatorname{Hom}\left(F / F_{0}, Z\right)=0$ where $F_{0}$ is a countable subgroup of $F$. Thus $A$ is not free.

THEOREM 4.3. Let $N$ be a nonempty proper subset of the primes. Then there is a locally free $B^{N}$-group that is not free.

Proof. Observe that the cotorsion completion of the group $I_{N}$ is a direct summand of $E$. Let $A$ be the group constructed in Theorem 4.2 with $U_{\alpha}=I_{N}$ for each $\alpha<\Omega$. Since Hom $\left(I_{N}, Z\right)=0$, we have that $A$ is a nonfree, locally free group. Note 
that $I_{N} \otimes A=\bigcup_{\alpha<\Omega}\left(I_{N} \otimes A_{\alpha}\right)$. The exact sequence $A_{\alpha} \gg A_{\alpha+1} \rightarrow I_{N}$ yields the exact sequence $I_{N} \otimes A_{\alpha} \longrightarrow I_{N} \otimes A_{\alpha+1} \rightarrow I_{N} \otimes I_{N}$. By a Theorem of Baer [2], it is easily seen that $I_{N} \otimes I_{N} \cong I_{N}$. Since, for each $\alpha, I_{N} \otimes A_{\alpha}$ is free as an $I_{N}$-module and since $\left(I_{N} \otimes A_{\alpha+1}\right) /\left(I_{N} \otimes A_{\alpha}\right) \cong I_{N}$, it follows that $I_{N} \otimes A$ is free as an $I_{N^{-}}$ module. By Theorem $3.4, A$ is a $B^{N}$-group.

COROLlaRY 4.4. If $\mu$ is an uncountable cardinal and if $N$ is a nonempty proper subset of the primes, then the completely decomposable group $\sum_{\mu} I_{N}$ contains a locally free group that is not completely decomposable.

Proof. We may assume that $\mu=\aleph_{1}$. Let $A$ be a $B^{N}$-group satisfying Theorem 4.3. Then the exact sequence $Z \longrightarrow I_{N} \rightarrow I_{N} / Z$ yields that $A \cong Z \otimes A \gg I_{N} \otimes A \cong \sum_{N_{1}} I_{N}$.

If the set $N$ consists of a single prime $p$, let $B^{(p)}$ denote $B^{N}$. Note that the statement that $G$ is a $B^{(p)}$-group for each prime $p$ does not imply the statement that $G$ is a Baer group. Indeed, in view of Corollary 3.2, Chase [5] constructed a group that is a $B^{(p)}$-group for each $p$ but that is not a Baer group. We also remark that if $G$ is a $B^{(p)}$-group for each $p$, then Corollary 3.5 implies that $G$ is torsion free. Our concluding theorem shows that if a group $G$ is a $B^{(p)}$-group for each prime $p$ then, for any torsion group $T$, a quasi-splitting extension of $T$ by $G$ is necessarily a splitting extension.

THEOREM 4.5. If $G$ is a $B^{(p)}$-group for each prime $p$, then $\operatorname{Ext}(G, T)$ is torsion free for any torsion group $T$.

Proof. Let $p$ be a prime. Then $T=H+T_{p}$ where $H_{p}=0$. Therefore, $\operatorname{Ext}(G, T)$ $\cong \operatorname{Ext}(G, H)+\operatorname{Ext}\left(G, T_{p}\right)=\operatorname{Ext}(G, H)$ since $\operatorname{Ext}\left(G, T_{p}\right)=0$. By a theorem in [4], we have the isomorphism

$$
\begin{array}{r}
\text { Hom }(C(p), \operatorname{Ext}(G, H))+\operatorname{Ext}(C(p), \operatorname{Hom}(G, H)) \\
\cong \operatorname{Ext}(C(p) \otimes G, H)+\operatorname{Hom}(\operatorname{Tor}(C(p), G), H)
\end{array}
$$

where $C(p)$ is the cyclic group of order $p$. Since $C(p) \otimes G$ is $p$-primary and since $H_{p}=0$, then Ext $(C(p) \otimes G, H)=0$. Also Hom (Tor $\left.(C(p), G), H\right)=0$ since $G$ is torsion free. Thus $\operatorname{Hom}(C(p), \operatorname{Ext}(G, T)) \cong \operatorname{Hom}(C(p)$, Ext $(G, H))=0$. Since $p$ was an arbitrary prime, it follows that $\operatorname{Ext}(G, T)$ is torsion free.

\section{REFERENCES}

1. R. Baer, The subgroup of the elements of finite order of an Abelian group, Ann. of Math. 37 (1936), 766-781.

2. - Abelian groups without elements of finite order, Duke Math. J. 3 (1937), 68-112.

3. - Die Torsionsuntergruppe einer Abelschen Gruppe, Math. Ann. 135 (1958), 219-234.

4. H. Cartan and S. Eilenberg, Homological algebra, Princeton Univ. Press, Princeton, N. J., 1956.

5. S. Chase, Function topologies on Abelian groups, Illinois J. Math. 7 (1963), 593-608.

6. - "On group extensions and a problem of J. H. C. Whitehead," in Topics in Abelian groups, Scott, Foresman and Co., Chicago, Ill., 1963, pp. 173-193. 
7. J. Erdös, On the splitting problem of mixed Abelian groups, Publ. Math. Debrecen 5 (1958), 364-367.

8. L. Fuchs, Abelian groups, Hungarian Academy of Sciences, Budapest, 1958.

9. D. K. Harrison, Infinite Abelian groups and homological methods, Ann. of Math. 69 (1959), 366-391.

10. P. Hill and C. Megibben, Extending automorphisms and lifting decompositions in Abelian groups, Math. Ann. 175 (1968), 159-168.

11. R. J. Nunke, Slender groups, Acta. Sci. Math. Szeged 23 (1962), 67-73.

12. J. Rotman, On a problem of Baer and a problem of Whitehead in Abelian groups, Acta. Math. Acad. Sci. Hungar. 12 (1961), 245-254.

13. E. Specker, Additive gruppen von Folgen ganzer Zahlen, Portugal. Math. 9 (1950), 131-140.

14. C. Walker, Properties of Ext and quasi-splitting of Abelian groups, Acta. Math. Acad. Sci. Hungar. 15 (1964), 157-160.

UNIVERSITY OF HOUSTON, Houston, TeXas 